\title{
Tumor Free
}

National Cancer Institute

\section{Source}

National Cancer Institute. Tumor Free. NCI Thesaurus. Code C156848.

There is no evidence of a tumor in the individual. 